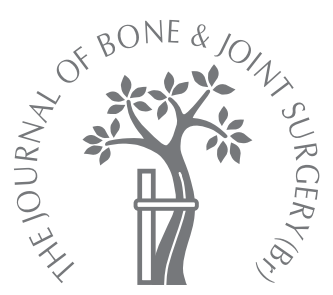

H. Niki,

T. Hirano,

H. Okada,

M. Beppu

From St. Marianna

University School of

Medicine, Kanagawa, Japan

H. Niki, MD, PhD, Associate

Professor

- T. Hirano, MD, PhD,

Orthopaedic Surgeon

H. Okada, MD, Orthopaedic

Surgeon

- M. Beppu, MD, PhD,

Professor and Chairman

Department of Orthopaedic

Surgery

St. Marianna University School

of Medicine, 2-16-1 Sugao,

Miyamae-ku, Kawasaki,

Kanagawa 216-8511, Japan.

Correspondence should be sent to $\mathrm{Dr}$ H. Niki; e-mail:

h2niki@marianna-u.ac.jp

(ㄷ)2010 British Editorial Society of Bone and Joint Surgery

doi:10.1302/0301-620X.92B3.

$23186 \$ 2.00$

$J$ Bone Joint Surg [Br] 2010;92-B:380-6.

Received 31 July 2009;

Accepted after revision 19

November 2009

\title{
Combination joint-preserving surgery for forefoot deformity in patients with rheumatoid arthritis
}

Proximal osteotomies for forefoot deformity in patients with rheumatoid arthritis have hitherto not been described. We evaluated combination joint-preserving surgery involving three different proximal osteotomies for such deformities. A total of 30 patients (39 feet) with a mean age of $\mathbf{5 5 . 6}$ years (45 to 67) underwent combined first tarsometatarsal fusion and distal realignment, shortening oblique osteotomies of the bases of the second to fourth metatarsals and a fifth-ray osteotomy.

The mean follow-up was 36 months (24 to 68). The mean foot function index scores for pain, disability and activity subscales were 18,23 , and 16 respectively. The mean Japanese Society for Surgery of the Foot score improved significantly from 52.2 (41 to 68) to 89.6 (78 to 97). Post-operatively, 14 patients had forefoot stiffness, but had no disability. Most patients reported highly satisfactory walking ability. Residual deformity and callosities were absent. The mean hallux valgus and intermetatarsal angles decreased from $47.0^{\circ}$ $\left(20^{\circ}\right.$ to $\left.67^{\circ}\right)$ to $9.0^{\circ}\left(2^{\circ}\right.$ to $\left.23^{\circ}\right)$ and from $14.1^{\circ}\left(9^{\circ}\right.$ to $\left.20^{\circ}\right)$ to $4.6^{\circ}\left(1^{\circ}\right.$ to $\left.10^{\circ}\right)$, respectively. Four patients had further surgery including removal of hardware in three and a fifth-ray osteotomy in one.

With good peri-operative medical management of rheumatoid arthritis, surgical repositioning of the metatarsophalangeal joint by metatarsal shortening and consequent relaxing of surrounding soft tissues can be successful. In early to intermediate stages of the disease, it can be performed in preference to joint-sacrificing procedures.

Various operations for the correction of deformity of the forefoot in patients with rheumatoid arthritis have been described. ${ }^{1-5}$ There is a consensus that the function of the metatarsophalangeal (MTP) joint can be sacrificed in such patients. However, treatment of rheumatoid arthritis has improved dramatically with the introduction of diseasemodifying anti-rheumatic drugs and biological agents leading us to reconsider the benefits of joint preservation.

Previous studies on joint-preserving surgery for rheumatoid forefoot deformity have involved distal osteotomy of the lesser metatarsals. $^{2-5}$ We initially used distal osteotomy, but these procedures are technically demanding, especially in patients with severe dislocation or contracture. We therefore introduced a combination joint-preserving operation involving three different proximal shortening metatarsal osteotomies.

Our aim in this study was to assess the outcome of this combination joint-preserving surgery for forefoot deformity in patients with rheumatoid arthritis.

\section{Patients and Methods}

This retrospective study included 30 patients (39 feet) with rheumatoid arthritis in remission. There were 29 women and one man with a mean age at surgery of 55.6 years (45 to 67). All had severe pain, deformity, plantar callosities and mild or moderate destruction of the metatarsal heads. The interphalangeal joint of the hallux was healthy in all the patients and none had valgus deformity of the hindfoot. The mean follow-up was 36.6 months (24 to 68 ) when assessment by questionnaire and clinical and radiological examination was carried out.

Exclusion criteria included severe destruction of the metatarsal head, infection or ulceration of the forefoot and previous hindfoot or forefoot surgery.

Operative technique. The combination jointpreserving operation has three components as follows: a first tarsometatarsal (TMT) fusion and distal re-alignment (the modified Lapidus procedure with shortening), ${ }^{6}$ a shortening oblique osteotomy of the bases of the second to fourth metatarsals ${ }^{7}$ and osteotomy of the fifth 
ray (the modified Coughlin procedure, originally indicated for treating a bunionette with shortening). ${ }^{8}$

The size of the wedge to be removed from the base of the first metatarsal is determined pre-operatively. The proximal phalanx, metatarsal and tarsal bones in the midfoot are traced on a standing anteroposterior (AP) radiograph. Using these drawings, the first metatarsal is abducted and shortened so that the distal end of its head came in contact with the proximal end of the proximal phalanx and the metatarsal head is repositioned on the lateral sesamoid as the target position for correction. The amount of bone to be removed from the base of the first metatarsal can thereby be estimated.

Under general anaesthesia the contracted proximal interphalangeal (PIP) joints are carefully manipulated. The open operation always starts with the hallux. First the MTP joint is treated by a series of procedures comprising release of the distal soft tissues, shaving of the medial eminence, and plication of the medial capsule. Osteotomy is performed at the base of the first metatarsal and the joint surface of the medial cuneiform. A minimum amount of bone is removed from the cuneiform by an osteotomy parallel to its joint surface, and the amount which has been determined pre-operatively is removed from the metatarsal. The latter is then abducted, shortened, supinated and fixed temporarily to the cuneiform by a Kirschner (K-) wire. The metatarsal head is repositioned on the sesamoid, with the intermetatarsal angle of the first and second metatarsals being almost zero. The metatarsal is neither dorsiflexed or plantar flexed with its axis parallel to that of the second metatarsal on the lateral view which is confirmed by fluoroscopy. The metatarsal and medial cuneiform are fixed by a cannulated cortical screw $3.5 \mathrm{~mm}$ in diameter and another cannulated cortical screw $3 \mathrm{~mm}$ in diameter is inserted to stabilise the Lisfranc ligament complex, comprising the first and second metatarsals and the medial and intermediate cuneiforms.

For the shortening osteotomy of the second to fourth metatarsals, a dorsal longitudinal incision of approximately $2.5 \mathrm{~cm}$ is made between the bones of the second and third metatarsals with a further incision over the fourth metatarsal. Osteotomy is performed as obliquely as possible at an angle of more than $45^{\circ}$ to the longitudinal axis of the metatarsal. The distal fragment is displaced dorsally and proximally and fixed temporarily by the guide-wire for the cannulated screw. The second metatarsal is shortened so that the heads of the first and second metatarsals are aligned at almost the same level and this is checked by fluoroscopy. The osteotomy is fixed by a cannulated cortical screw $3 \mathrm{~mm}$ in diameter. The third and fourth metatarsals are similarly shortened and fixed to form a smooth arc towards the lateral side.

A further longitudinal incision is made, centered over the lateral aspect of the fifth metatarsal beginning at the midportion of the proximal phalanx and extending to a point $2 \mathrm{~cm}$ below the base of the fifth metatarsal. A diaphyseal osteotomy is performed from the lateral side, with the saw blade orientated transversely and superiorly. Therefore, with internal rotation of the distal fragment the metatarsal head moves superiorly, medially and proximally. A cannulated cortical screw, $3 \mathrm{~mm}$ in diameter is used for fixation. The dorsal capsule and collateral ligaments of each MTP joint are released. Any dislocation is reduced, and the MTP joints of the lesser toes are temporarily fixed by K-wires $1.2 \mathrm{~mm}$ in diameter.

Post-operatively, the foot is placed in a bulky dressing for one week followed by a well-padded short-leg cast for two weeks after which the wires are removed. Partial weightbearing in a short-leg plaster shell and range-of-movement exercises are encouraged. At seven weeks post-operatively, the shell is removed and full weight-bearing is allowed. The patient is instructed to wear adjustable shoes to accommodate an arch support until union of the osteotomies occurred.

Clinical assessment was according to the Japanese Society for Surgery of the Foot, foot and ankle scale which was developed in 2005 for subjective and objective clinical assessment of associated foot disease in rheumatoid arthritis. ${ }^{9}$ This scale has been verified for validity, reliability and responsiveness. ${ }^{10}$ Scores range from 0 to 100 points and cover five major items such as pain (30 points), deformity ( 25 points), movement (15 points), walking ability (20 points) and activities of daily living ( 10 points). The feet were also examined post-operatively for symptomatic plantar callosities and residual toe deformities.

Subjective assessment was by questionnaire using two methods, the foot function index, ${ }^{11}$ specific for disease and site and the comprehensive 36-item Short-Form health survey version 2 (SF-36v2). ${ }^{12}$ The index is validated and reliable for measuring foot pain and disability. For the eight SF-36v2 subscales, the mean score and SD were calculated by means of norm-based scoring.

The hallux valgus angle and intermetatarsal angles of the first and second metatarsals, the first and fifth metatarsals, and the fourth and fifth metatarsals were measured pre- and post-operatively on AP radiographs. The position of the head of the first metatarsal relative to its sesamoid and the relationship between the shortness of each metatarsal and the congruency of each MTP joint were also examined. From these results, the parameters by which good repositioning was achieved and maintained were estimated. The classification of Larsen, Dale and Eek ${ }^{13}$ was used to assess the severity of destruction of the metatarsal head. Grade I or grade II indicated mild destruction and grade III moderate destruction.

Statistical analysis. The data were analysed statistically by SPSS version 5 software (SPSS Inc., Chicago, Illinois). The pre- and post-operative Japanese Society for Surgery of the Foot scores and radiological variables were analysed by the paired $t$-test and differences were considered to be significant at a $\mathrm{p}$-value $\leq 0.05$.

\section{Results}

The mean size of shortening of the first metatarsal was $3.4 \mathrm{~mm}$ (1.0 to 5.0 ) on the medial and $5.9 \mathrm{~mm}$ (3.0 to 10.0) on the lateral side. The mean Japanese Society score improved significantly from 52.2 points (41 to 68 ) pre-operatively to 89.6 points (78.0 to 97.0 ) post-operatively (paired $t$-test, 


\begin{tabular}{|c|c|c|}
\hline & Pre-operative & Post-operative \\
\hline General pain (30 points) & $14.0(10$ to 20$)$ & 29.3 (20 to 30$)$ \\
\hline \multicolumn{3}{|l|}{ Deformity ( 25 points) } \\
\hline \multicolumn{3}{|l|}{ Forefoot } \\
\hline Hallux & $0.8(0$ to 5$)$ & 4.7 (3 to 5$)$ \\
\hline Lesser toes & $0.2(0$ to 1$)$ & 4.9 (3 to 5$)$ \\
\hline Midfoot & $4.9(3$ to 5$)$ & 4.9 (3 to 5$)$ \\
\hline Hindfoot & $10.0(10$ to 10$)$ & $10.0(10$ to 10$)$ \\
\hline \multicolumn{3}{|l|}{ Movement (15 points) } \\
\hline Forefoot (MTP/IP* joints) & $0.8(0$ to 3$)$ & $2.4(0$ to 3$)$ \\
\hline Hindfoot & 9.7 (5 to 10$)$ & $9.7(5$ to 10$)$ \\
\hline Walking ability (20 points) & $8.7(5$ to 10$)$ & $18.0(10$ to 20$)$ \\
\hline \multicolumn{3}{|l|}{ Activities of daily living (10 points) } \\
\hline Climbing or descending stairs & $0.9(0$ to 1$)$ & 1.1 (1 to 2$)$ \\
\hline Sitting on heels & $0.5(0$ to 1$)$ & $0.8(0$ to 2$)$ \\
\hline Standing on toes & $0.6(0$ to 1$)$ & 1.6 (1 to 2$)$ \\
\hline Footwear & $0.7(0$ to 1$)$ & 1.7 (1 to 2$)$ \\
\hline Japanese-style toilet & $0.4(0$ to 1$)$ & $0.5(0$ to 1$)$ \\
\hline Total & 52.2 (41 to 68$)$ & 89.6 (78 to 97 ) \\
\hline
\end{tabular}

$\mathrm{p}<0.001$; Table I). Any symptomatic plantar callosities seen pre-operatively disappeared in all patients. In one foot in which the fifth metatarsal had not been shortened, new callus developed beneath its head and this was resolved later using the modified Coughlin technique. Figure 1 shows the pre- and post-operative clinical and radiological appearance.

The mean total foot function index score was 19.3 (16.6 to 23.2). Mean scores for pain, disability and activity subscales were 18 (14.2 to 29.6), 23 (4.2 to 59.3), and 16 (2.1 to 34.7), respectively. A total of 14 patients recorded some stiffness of the forefoot post-operatively, but reported no disability. Most had highly satisfactory walking ability.

The mean scores and SDs for the eight SF-36v2 subscales calculated by the norm-based scoring method were as follows: 34.9 (2.3 to 55.1) for physical functioning, 32.4 (1.7 to 46.0) for physical role, 39.5 (22.5 to 61.4$)$ for bodily pain, 35.6 (18.1 to 48.9 ) for general health, 38.7 (31.8 to 56.4) for vitality, 39.8 (11.1 to 57.1 ) for social functioning, 43.8 (31.1 to 56.6) for emotional role and 43.8 (38.5 to 54.4) for mental health.

The mean hallux valgus angle and the intermetatarsal angle of the first and second metatarsals decreased significantly from $47.0^{\circ}\left(20^{\circ}\right.$ to $\left.67^{\circ}\right)$ to $9.0^{\circ}\left(2^{\circ}\right.$ to $\left.23^{\circ}\right)$ and from $14.1^{\circ}\left(9^{\circ}\right.$ to $\left.20^{\circ}\right)$ to $4.6^{\circ}\left(1^{\circ}\right.$ to $\left.10^{\circ}\right)$, respectively (paired $t$-test, $\mathrm{p}<0.001$, Table II). In patients who showed favourable repositioning during follow-up, the head of each metatarsal was repositioned to the level of the proximal part of each dislocated proximal phalanx on pre-operative radiographs (Fig. 2).

Skin problems localised to the lateral sides of the second and third toes were observed in five feet post-operatively, but resolved with removal of the K-wires. Delayed wound healing occurred in five feet. Patients with these complications had undergone surgery early in our series. Those with moderate pre-operative destruction of the metatarsal heads (Larsen grade III) tended to have restricted movement of the MTP joint post-operatively. None of the patients reported recurrence of deformity of the toe although those with severe contracture of the PIP joints pre-operatively had a slightly restricted range of movement in these joints post-operatively. There was no case of nonunion, recurrence of deformity, formation of callosity or infection. Four patients had reoperations including removal of hardware in three feet and an osteotomy of the fifth ray for a residual callosity beneath the head of the fifth metatarsal.

\section{Discussion}

Arthrodesis and resection are the two major surgical options for the first MTP joint in reconstruction of the rheumatoid forefoot. However, there are several drawbacks to these traditional procedures. In the literature success rates of good to excellent for resection vary from $51 \%$ to $93 \%{ }^{14-17}$ and the major problems have been high recurrence rates for hallux valgus, metatarsalgia and plantar callosities, by as much as $53 \%, 36 \%$ and $61 \%$, respectively. ${ }^{14,15,18}$ In the 1970 s it was noted that a stiff first MTP joint seemed to produce a better outcome $^{19,20}$ and fusion grew in popularity with high degrees of patient satisfaction. ${ }^{1,21,22}$ The cosmetic appearance was improved $^{23}$ and, using the Harris foot mat, the hallux was shown to bear weight in $50 \%$ more cases after fusion than after Keller's operation. ${ }^{24}$ However, the alignment of the hallux is important, but can be difficult to achieve. ${ }^{18,25}$ The rate of pseudarthrosis varies from $0 \%$ to $44 \%^{1,17,26}$ and this may require re-operation. ${ }^{27}$ Inter-phalangeal joint degeneration can develop after arthrodesis. ${ }^{14,23,25} \mathrm{~A}$ randomised study showed no significant differences in outcome and patient satisfaction between resection and arthrodesis. ${ }^{28}$ Also, there was 


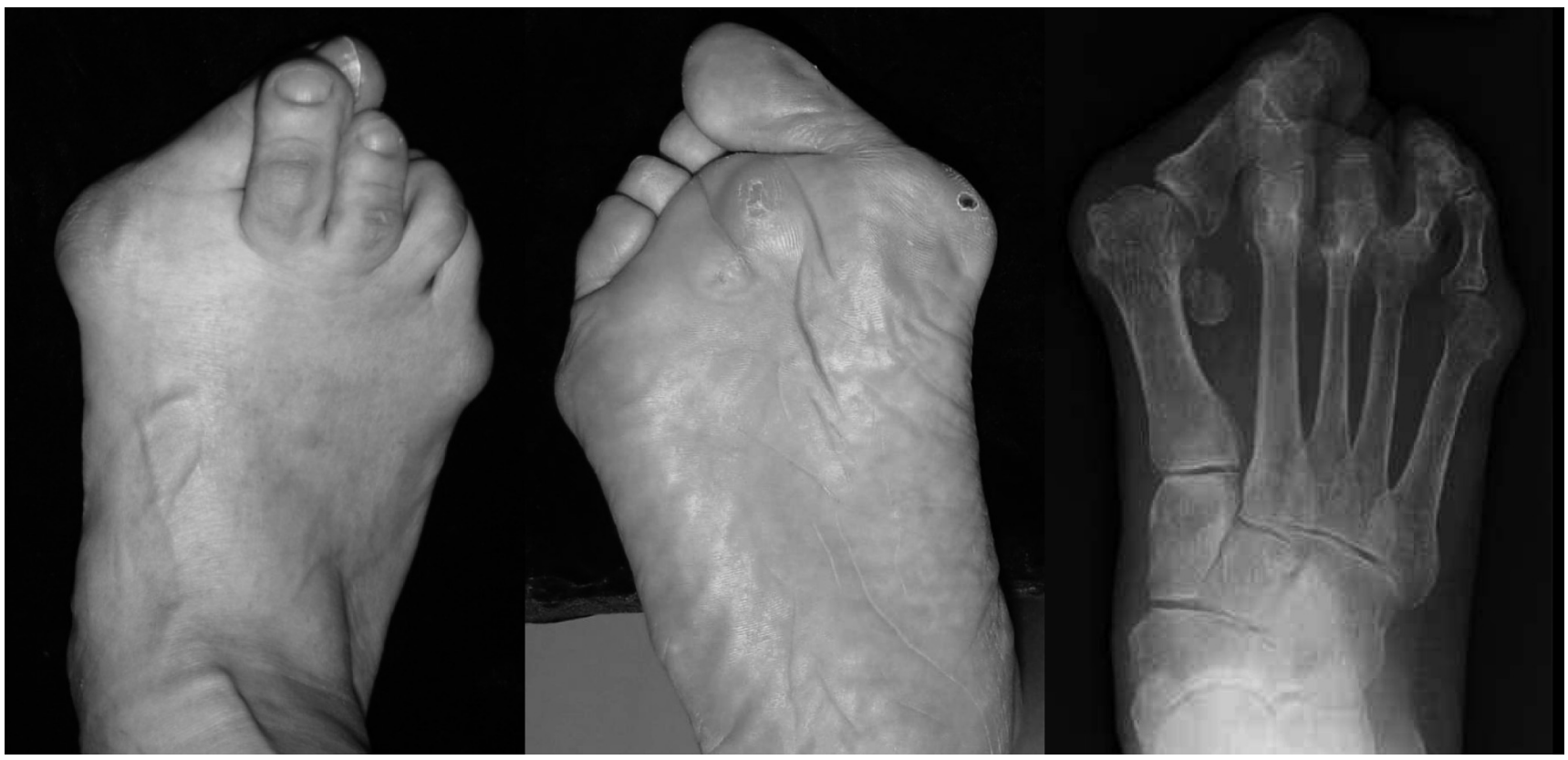

Fig. 1a

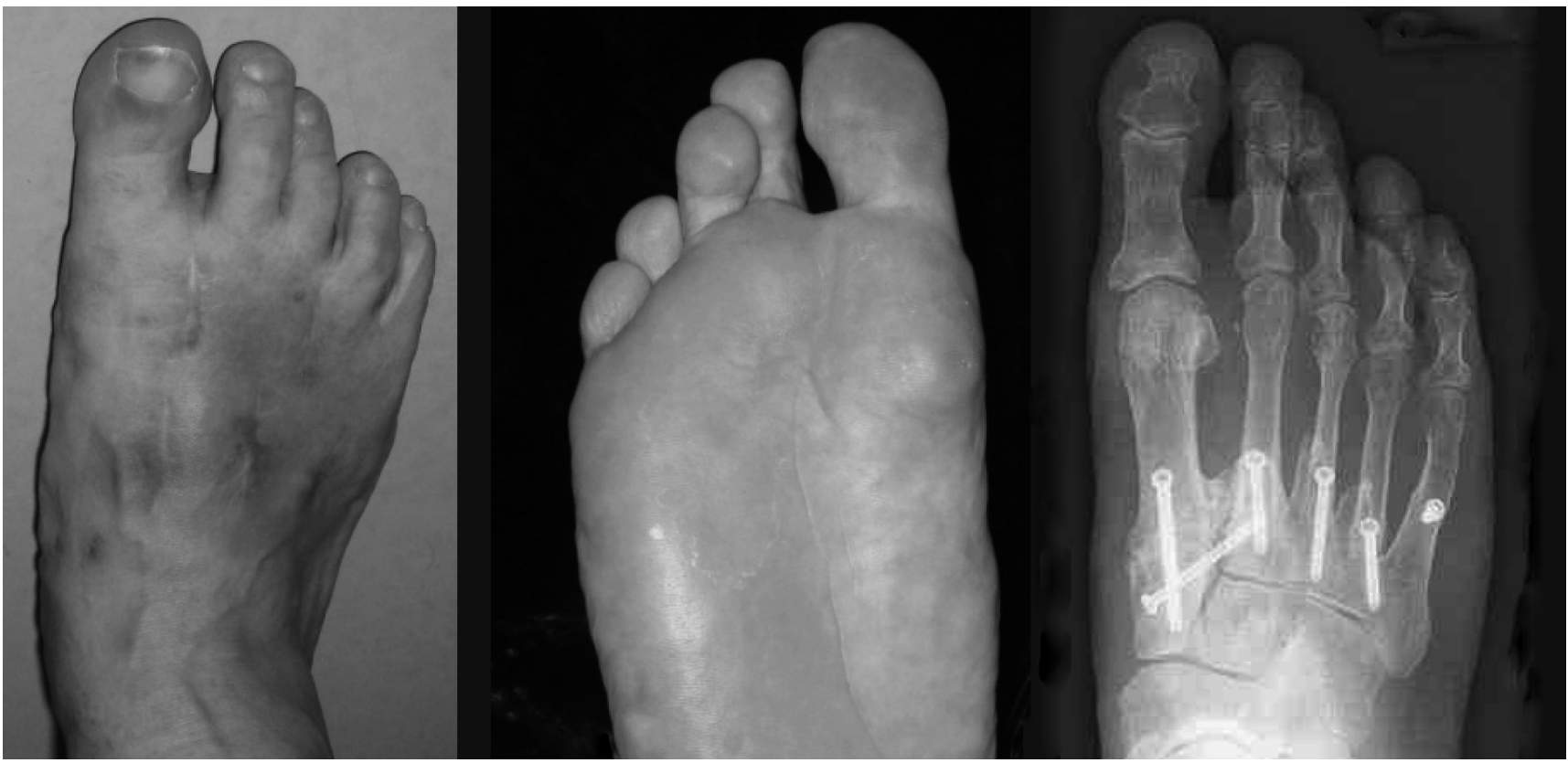

Fig. 1b

Figure 1a - Pre-operative photographs and anteroposterior (AP) radiograph of a 48-year-old woman with rheumatoid arthritis. The Japanese Society for Surgery of the Foot (JSSF) score was 48 points with difficulty in walking because of painful plantar callosities. The hallux valgus angle was $62^{\circ}$ and the intermetatarsal angle of the first and second metatarsals $18^{\circ}$. Figure $1 \mathrm{~b}$ - Post-operative photographs and AP radiograph at 30 months showing favourable joint congruency in the hallux and lesser toes, with no recurrence of the deformity or callosities. The JSSF score was 97 points. The hallux valgus angle had decreased to $6^{\circ}$ and the intermetatarsal angle of the first and second metatarsals to $6^{\circ}$.

no significant difference in the foot function index scores and frequency of callosities between the treatments. ${ }^{28}$ When these findings were compared with our results, no difference was found in the index score, but plantar callosities and residual deformity were absent post-operatively in our study. This showed that the joint-preserving operation was more effective for forefoot callosities and recurrence of deformity than joint-sacrificing procedures.

Symptomatic plantar callosities are the main reason for seeking surgical treatment in rheumatoid deformity of the 
Table II. Mean (SD) pre- and post-operative radiological measurements

\begin{tabular}{clcc}
\hline & Pre-operative & Post-operative & p-value \\
\hline Hallux valgus angle $\left(^{\circ}\right)$ & $47.0(16.3)$ & $9.0(6.2)$ & $<0.001^{*}$ \\
Intermetatarsal angle $\left(^{\circ}\right)$ & & & \\
1st and 2nd metatarsals & $14.1(3.8)$ & $4.6(3.2)$ & $<0.001^{*}$ \\
1st and 5th metatarsals & $34.7(3.7)$ & $22.3(5.6)$ & $<0.001^{*}$ \\
4th and 5th metatarsals & $10.5(3.0)$ & $9.1(4.1)$ & $<0.001^{*}$ \\
\hline
\end{tabular}

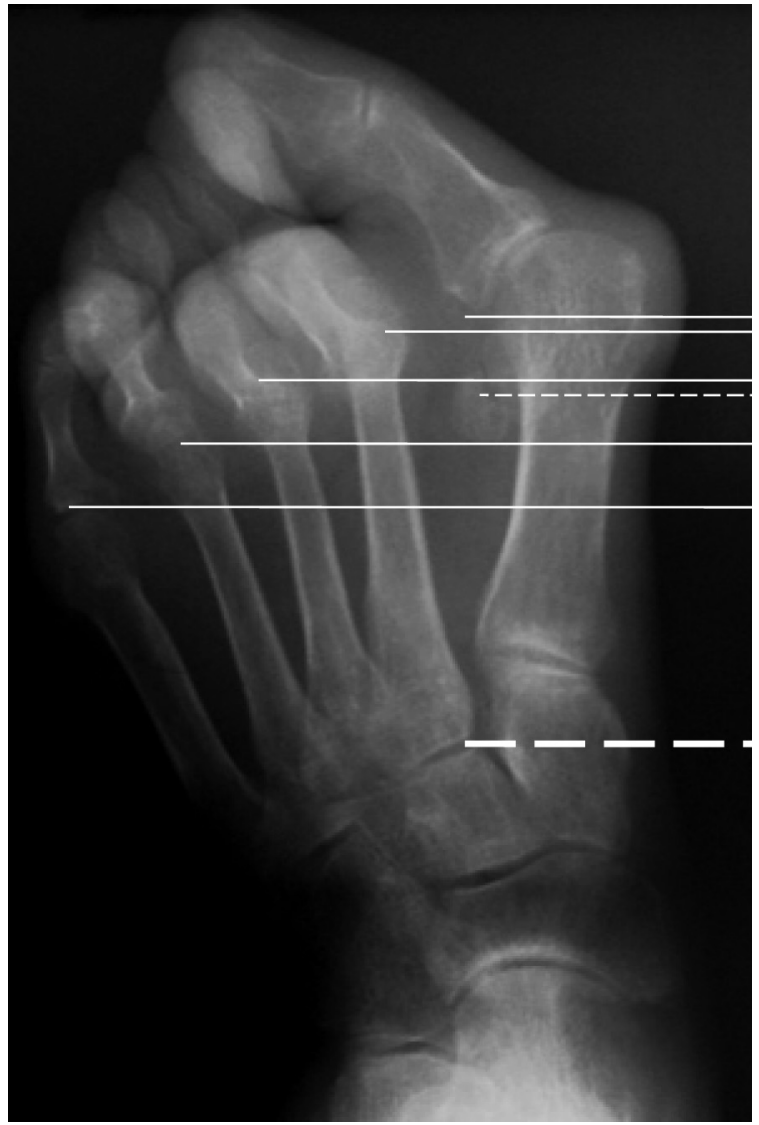

Fig. 2a

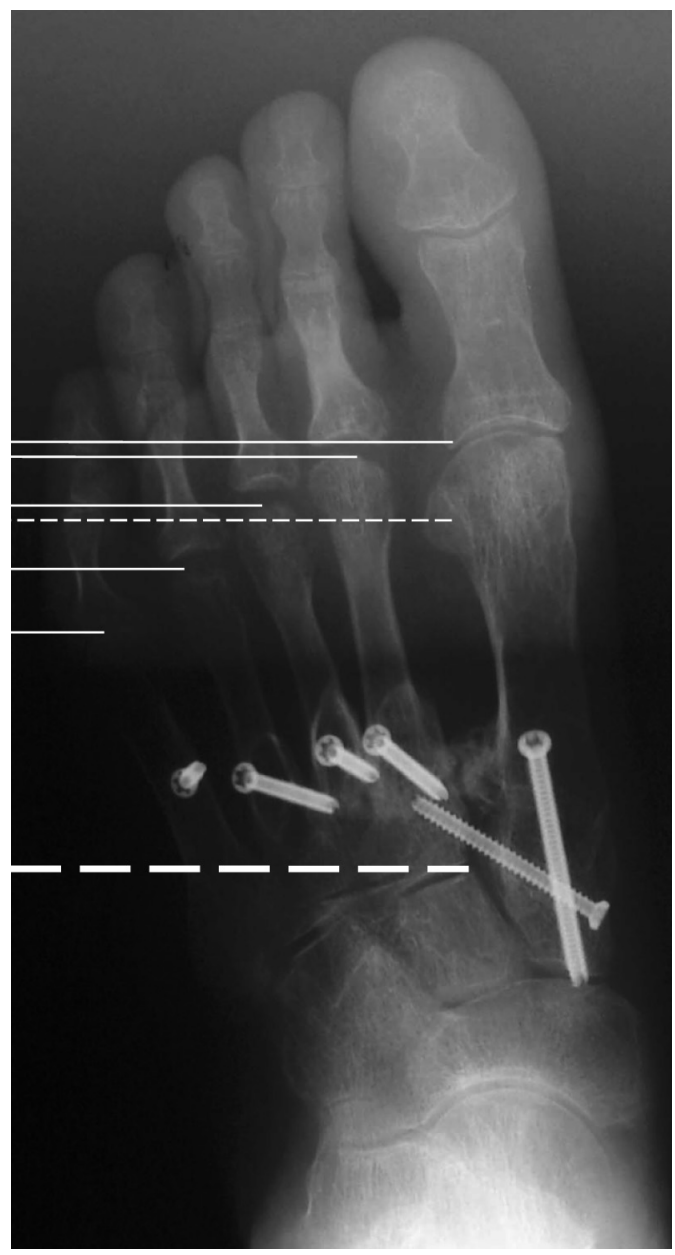

Fig. $2 b$

Radiographs taken pre-operatively (a) and at the final follow-up at 31 months (b) showing straight lines drawn between the intermediate cuneiforms (thick dotted line) and between the lateral sesamoids (thin dotted line). This ensures that the images were aligned at the same level. The heads of each metatarsal were repositioned to the levels of the proximal parts of each dislocated proximal phalanx (solid lines).

forefoot. ${ }^{1}$ Formation of callosities is not related directly to the presence or absence of synovitis and correlates poorly with the degree of bone destruction. ${ }^{3}$ Instead, loss of joint function because of dislocation of the proximal phalanges is thought to be the primary cause of such formation. ${ }^{3}$ The preservation of joint function is therefore important to prevent recurrence of callosities. Laroche et $\mathrm{al}^{29}$ showed that in patients with inactive rheumatoid arthritis, stiffness of the
MTP joints was related to a decrease in walking speed and stride length. These findings support the hypothesis that preservation of MTP joint function improves gait in patients with rheumatoid arthritis.

There have been several studies on joint-preserving surgery for the treatment of forefoot deformity in patients with rheumatoid arthritis, all of which have involved osteotomy of the metatarsals. ${ }^{2-4}$ However, in patients with severe dis- 
location or contracture, exposure of joints and repositioning can be performed more easily after an osteotomy, because metatarsal shortening causes relaxation of periarticular soft tissues. Distal osteotomy remains unstable unless it is internally fixed, making joint exposure and repositioning difficult. Furthermore, the procedure requires a high level of skill and is associated with the risk of necrosis of the metatarsal heads. We therefore performed shortening osteotomy at the bases of the metatarsals. When considering the anatomical features of each metatarsal and their influence on forefoot deformity, we combined three different osteotomies. The reason for adopting first TMT fusion and distal realignment (the modified Lapidus procedure) was that simultaneous correction of metatarsus varus with some pronation and shortening of the metatarsal were possible. Our pre-operative drawings set the positions of the base of the proximal phalanx of the hallux and the lateral sesamoid as the target positions for correction for the following reasons. First, restriction of extension of the MTP joint because of contracture of flexor hallux brevis can be prevented if a favourable position between the head of the metatarsal and the sesamoid is achieved by metatarsal shortening. Secondly, repositioning of the metatarsal head on the sesamoid results in stabilisation of the MTP joint and thus prevents recurrence of the deformity.

Oblique shortening basal osteotomy of the second to fourth metatarsals was undertaken for three reasons. First, in comparison with distal osteotomy, basal osteotomy allowed easy exposure and reduction of the MTP joint by means of rigid internal fixation. Dissection of the surrounding soft tissue could be minimised, thereby preventing postoperative joint contracture. Secondly, at operation, it is easy to adjust the position of the metatarsal heads in relation to each other. Finally, shortening and elevation of the metatarsal heads were possible simultaneously.

Barouk and Barouk ${ }^{2}$ proposed a modified Weil osteotomy in which the metatarsal head was elevated. However, it is very difficult to make two osteotomy cuts (double-layer osteotomy) at a small angle from the head to the neck, which is small and thin. Moreover, the technique risks necrosis to the metatarsal head. Conversely, oblique proximal osteotomy requires only one cut. The Weil osteotomy ${ }^{2}$ may be biomechanically more advantageous than our method because, with the direction of our osteotomy, a weight-bearing load exerted on the metatarsal head can cause distal fragments to move dorsally. ${ }^{30}$ Nevertheless, by rigid internal fixation and a strict post-operative regimen, we have not experienced any case of nonunion, probably because the area of surface contact of our osteotomy was greater than that with the Weil osteotomy.

A fifth-ray osteotomy (the modified Coughlin procedure) was adopted for three reasons. First, an osteotomy can be easily performed from the lateral side. Also, with the saw blade orientated not only transversely, but also superiorly, the metatarsal head can be elevated at the same time that it is internally rotated. ${ }^{8} \mathrm{~A}$ large surface contact area and rigid internal fixation are helpful for bony union. Secondly, simultaneous shortening, elevation and internal rotation of the metatarsal head are possible. Finally, it is easy to adjust the position of the metatarsal head intra-operatively.

Several technical issues are important. First, all tissues must be treated gently to prevent ischaemic change. Secondly, when fixing the second to the fourth metatarsals, the pre- and post-operative axes of the bones must correspond since displacement of these axes may result in varus or valgus deformity of the toes. Finally, any dislocation of the MTP joint must be reduced to prevent the recurrence of callosity and soft-tissue adhesion on the plantar side of the metatarsal head in order to allow plantar flexion of the joint post-operatively. It is also important to confirm that after shortening, the metatarsal head is located proximally to the callosity and that no metatarsal head protrudes more than another on the plantar side.

Retrospective analysis of pre-operative radiographs showed that the most proximal parts of the bases of the dislocated proximal phalanges of the lesser toes were at almost the same level as the bases of the proximal phalanx of the hallux and lateral sesamoid. In patients who maintained favourable repositioning of the MTP joint, the heads of each metatarsal were repositioned to the levels of the proximal parts of each dislocated proximal phalanx. These findings support our opinion that repositioning of the heads of all metatarsals thereby creating a favourable environment for the MTP joint, results in a stable long-term outcome. With good peri-operative medical management of rheumatoid arthritis, surgical repositioning of the MTP joint by metatarsal shortening and consequent relaxation of surrounding soft tissues should be successful.

Barouk and Barouk ${ }^{2}$ performed tendon lengthening in all their patients. With severe dislocation, insufficient metatarsal shortening leaves the extensor tendons and intrinsic muscles under strain, leading to poor metatarsal repositioning. Although tendon lengthening is required in such cases, most patients in our study did not require this, probably because relaxation of the surrounding soft tissues was achieved by adequate metatarsal shortening. Obtaining favourable joint congruency without tendon lengthening is valuable for joint movement and would also influence gait significantly. ${ }^{29}$

There are several drawbacks to our procedure. First, it requires more time and is technically more demanding than resection arthroplasty. Secondly, it involves more incisions than distal osteotomy and is therefore more invasive. Therefore we do not advise the operation in the elderly. Thirdly, temporary fixation of the MTP joints by K-wires may cause a stiffness and the use of temporary fixation is ongoing from our technique of resection arthroplasty. As used by Barouk and Barouk, ${ }^{2}$ temporary fixation up to the PIP joint may better prevent stiffness of the MTP joint.

Severe destruction of the metatarsal head is a contraindication to this procedure. When this is severe, there are few benefits from joint preservation, and traditional arthro- 
desis or resection arthroplasty is recommended for advanced arthritis of the MTP joint. This decision would be made on the basis of the pre-operative radiological or CT findings.

In conclusion, joint-preserving surgery by a modified Lapidus procedure and oblique shortening basal osteotomy of the lesser metatarsals is beneficial for correcting forefoot deformities in patients with early or intermediate rheumatoid arthritis. Preservation of the MTP joint by proximal osteotomy as opposed to resection arthroplasty can be expected to give better correction, function and appearance.

No benefits in any form have been received or will be received from a commercial party related directly or indirectly to the subject of this article.

\section{References}

1. Coughlin MJ. Rheumatoid forefoot reconstruction: a long-term follow-up study. $J$ Bone Joint Surg [Am] 2000;82-A:322-41.

2. Barouk LS, Barouk P. Joint-preserving surgery in rheumatoid forefoot: preliminary study with more-than-two-year follow-up. Foot Ankle Clin 2007;12:435-54

3. Hanyu T, Yamazaki H, Murasawa A, Tohyama C. Arthroplasty for rheumatoid forefoot deformities by a shortening oblique osteotomy. Clin Orthop 1997;338:131-8.

4. Helal B, Greiss M. Telescoping osteotomy for pressure metatarsalgia. J Bone Joint Surg [Br] 1984;66-B:213-17.

5. Nagashima M, Kato K, Miyamoto Y, Takenouchi K. A modified Hohman method for hallux valgus and telescoping osteotomy for lesser toe deformities in patients with rheumatoid arthritis. Clin Rheumatol 2007;26:39-43.

6. Lapidus PW. The author's bunion operation from 1931 to 1959. Clin Orthop 1960;16:119-35.

7. Okuda R, Kinoshita M, Morikawa J, Jotoko T, Abe M. Surgical treatment for hallux valgus with painful plantar callosities. Foot Ankle Int 2001;22:203-8.

8. Mann RA, Coughlin MJ. Keratotic disorders of the plantar skin. In: Coughlin MJ, Mann RA, eds. Surgery of the foot and ankle. Seventh ed. Vol. 1. St. Louis: Mosby, 2000:392-436.

9. Niki H, Aoki H, Inokuchi S, et al. Development and reliability of a standard rating system for outcome measurement of foot and ankle disorders. II: interclinician and intraclinician reliability and validity of the newly established standard rating scales and Japanese Orthopaedic Association rating scale. J Orthop Sci 2005;10:466-74.

10. Niki H, Aoki H, Inokuchi S, et al. Development and reliability of a standard rating system for outcome measurement of foot and ankle disorders. I: development of standard rating system. J Orthop Sci 2005;10:457-65.

11. Budiman-Mak E, Conrad KJ, Roach KE. The foot function index: a measure of foot pain and disability. J Clin Epidemiol 1991;44:561-70.

12. Fukuhara S, Suzukamo Y. Manual of SF-36v2 Japanese version. Institute for health Outcomes \& Process Evaluation Research, Kyoto, 2004.
13. Larsen A, Dale K, Eek M. Radiographic evaluation of rheumatoid arthritis and related conditions by standard reference films. Acta Radiol Diagn (Stockh) 1977;18:481-91.

14. Vahvanen V, Piiraienen $\mathbf{H}$, Kettunen P. Resection arthroplasty of the metatarsophalangeal joints in rheumatoid arthritis: a follow-up study of 100 patients. Scand J Rheum 1980;9:257-65

15. McGarvey SR, Johnson KA. Keller arthroplasty in combination with resection arthroplasty of the lesser metatarsophalangeal joints in rheumatoid arthritis. Foot Ankle 1988;9:75-80.

16. Dereymaeker G, Mulier T, Stuer P, Peeraer L, Fabry G. Pedodynographic measurements after forefoot reconstruction in rheumatoid arthritis patients. Foot Ankle Int 1997;18:270-6.

17. Vandeputte G, Steenwerckx A, Mulier T, Peeraer L, Dereymaeker G. Forefoot reconstruction in rheumatoid arthritis patients: Kellier-Lelievre-Hoffmann versus arthrodesis MTP1-Hoffmann. Foot Ankle Int 1999;20:438-43.

18. Hamalainen M, Raunio P. Long-term follow up of rheumatoid forefoot surgery. Clin Orthop 1997:340:34-8.

19. Watson MS. A long term follow-up of forefoot arthroplasty. J Bone Joint Surg [Br] 1974;56-B:527-33.

20. Mann RA, Oates JC. Arthrodesis of the first metatarsophalangeal joint. Foot Ankle 1980;1:159-66.

21. Newman RJ, Fitton JM. Conservation of metatarsal heads in surgery of rheumatoid arthritis of the forefoot. Acta Orthop Scand 1983;54:417-21

22. Mann RA, Schakel ME 2nd. Surgical correction of rheumatoid forefoot deformities Foot Ankle 1995;16:1-6.

23. Beauchamp CG, Kirby T, Rudge SR, Worthington BS, Nelson J. Fusion of the first metatarsophalangeal joint in forefoot arthroplasty. Clin Orthop 1984;190:249-53.

24. Henry AP, Waugh W, Wood H. The use of footprints in assessing the results of operations for hallux valgus: a comparison of Keller's operation and arthrodesis. $J$ Bone Joint Surg [Br] 1975;57-B:478-81.

25. Coughlin MJ, Abdo RV. Arthrodesis of the first metatarsophalangeal joint with Vitallium plate fixation. Foot Ankle 1994;15:18-28.

26. O'Doberty DP, Lowrie IG, Magnussen PA, Gregg PJ. The management of the painful first metatarsophalangeal joint in the older patient: arthrodesis or Keller's arthroplasty. J Bone Joint Surg [Br] 1990;72-B:839-42.

27. Hughes J, Grace D, Clark P, Klenerman L. Metatarsal head excision for rheumatoid arthritis: 4-year follow-up of 68 feet with \& without hallux fusion. Acta Orthop Scand 1991;62:63-6.

28. Grondal L, Brostrom E, Wretenberg P, Stark A. Arthrodesis versus Mayo resection: the management of the first metatarsophalangeal joint in reconstruction of the rheumatoid forefoot. J Bone Joint Surg [Br] 2006;88-B:914-19.

29. Laroche D, Pozzo T, Ornetti P, Tavernier C, Maillefert JF. Effects of loss of metatarsophalangeal joint mobility on gait in rheumatoid arthritis patients. Rheumatology 2006:45:435-40.

30. Trnka HJ, Mühlbauer M, Zettle R, et al. Comparison of the results of the Weil and Helal osteotomies for the treatment of metatarsalgia secondary to dislocation of the lesser metatarsophalangeal joints. Foot Ankle Int 1999;20:72-9 